\title{
Nucleated Growth of Iron Pyrite on Highly Oriented Pyrolytic Graphite (HOPG) by Chemical Vapor Deposition (CVD)
}

\author{
Yon Joo Kwon $^{1}$, John C. Hemminger ${ }^{1}$ \\ ${ }^{1}$ Department of Chemistry, University of California, Irvine CA 92697 USA
}

Iron pyrite, one of the phases in the $\mathrm{FeS}_{2}$ system, is a promising semiconductor for use in solar cell applications due to its earth-abundance, high absorption coefficient, and suitable bandgap. However, currently the efficiency of iron pyrite solar cell device does not exceed 3\% [1]. Many studies attempted to understand the cause of the low open-circuit voltage in pyrite devices. Some studies suggested that this is due to the naturally occurring metallic FeS-like phase impurities such as marcasite, pyrohotite, or troilite [2]. Other studies have demonstrated that sulfur and iron vacancies at the surface can cause the low open-circuit voltage [3]. Despite many efforts have been made to improve the efficiency of pyrite, it is incompletely understood how to preferentially grow solely pyrite. Thus, it is essential to understand the basic fundamental mechanisms of pyrite growth. As one of the methods, we examined the growth of pyrite particles in the presence of nucleation sites.

Here we studied chemical vapor deposition (CVD) of $\mathrm{FeS}_{2}$ on HOPG substrate treated by different methods. We report a direct correlation between the pre-history of the substrate and the nucleation growth of $\mathrm{FeS}_{2}$ by scanning electron microscopy (SEM). Previously, we were able to fabricate linearly ordered iron oxide nanoparticle arrays on HOPG. By using these iron oxide nanoparticle arrays as a starting material, we prepared two different pre-treated samples prior to CVD studies by introducing different sulfur environment. The morphology of nanoparticle arrays that were treated by H2S (g) was different to that of elemental sulfur treated sample. In addition, a comparison of different sulfur-gases used for the annealing process indicates a preference of site selective pyrite growth over other $\mathrm{FeS}_{2}$ phase growth. A blank HOPG and elemental sulfur treated HOPG were also prepared prior to CVD studies to observe the different growth morphologies of $\mathrm{FeS}_{2}$. Chemical tailoring of surface processes will help us understand and increase the efficiency of solar cell materials. 


\section{Reference:}

[1] Wadia, C.; Livisatos, A. P.; Kammen, D. M., Environ. Sci. Technol. 43 (2009,) p. 2072.

[2] Murphy, R.; Strongin, D. R., Surf. Sci. Rep. 64 (2009), p. 1.

[3] Birkholz, M.; Fiechter, S.; Hartmann, A.; Tributsch, H. Physical Review B, 43 (1991), p. 11926.
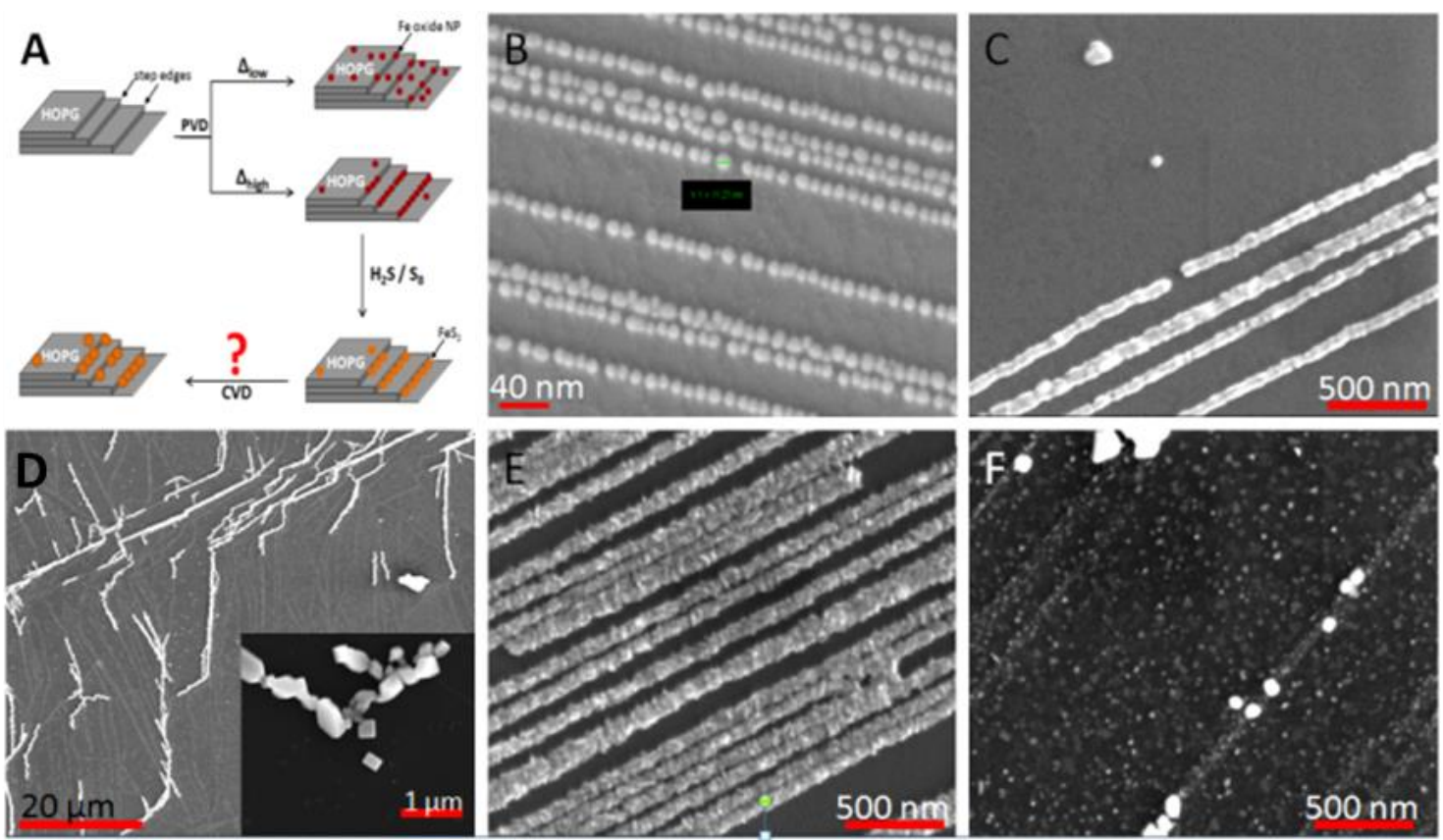

Figure 1: SEM images. (A) A schematic view of proposed research of Fe oxide deposited onto HOPG prior to CVD. (B) Iron oxide nanoparticles. (C) $\mathrm{H}_{2} \mathrm{~S}$ treatment on (B). (D) $\mathrm{S}_{8}$ treatment on (B). (E) Initial CVD deposition on (C). (F) Initial CVD deposition on (D). 